\title{
On the asymptotic behaviour of solutions of the stationary Navier-Stokes equations in dimension 3
}

\author{
Agathe Decaster ${ }^{a}$, Dragoş Iftimie ${ }^{\mathrm{b}, *}$ \\ a École Normale Supérieure de Lyon, Unité de Mathématiques Pures et Appliquées, UMR CNRS 5669, 46, allée d'Italie, 69364 Lyon Cedex 07, \\ France \\ ${ }^{\mathrm{b}}$ Université de Lyon, CNRS, Université Lyon 1, Institut Camille Jordan, 43 bd. du 11 novembre, Villeurbanne Cedex F-69622, France
}

Received 25 February 2015; received in revised form 17 November 2015; accepted 4 December 2015

Available online 11 December 2015

\begin{abstract}
In this paper, we address the problem of determining the asymptotic behaviour of the solutions of the incompressible stationary Navier-Stokes system in the full space, with a forcing term whose asymptotic behaviour at infinity is homogeneous of degree -3 . We identify the asymptotic behaviour at infinity of the solution. We prove that it is homogeneous and that the leading term in the expansion at infinity uniquely solves the homogeneous Navier-Stokes equations with a forcing term which involves an additional Dirac mass. This also applies to the case of an exterior domain.
\end{abstract}

(c) 2015 Elsevier Masson SAS. All rights reserved.

Keywords: Incompressible Navier-Stokes equations; Stationary; Asymptotic behaviour

\section{Introduction}

We consider the incompressible stationary Navier-Stokes equations with a forcing term in $\mathbb{R}^{3}$ :

$$
-\Delta U+(U \cdot \nabla) U+\nabla p=f, \quad \operatorname{div} U=0 \text { in } \mathbb{R}^{3}, \quad \lim _{|x| \rightarrow \infty} U(x)=0 .
$$

The forcing $f$ is given and the unknowns are the velocity field $U$ and the scalar pressure $p$. Clearly $p$ is uniquely (up to a constant) determined by $f$ and $U$. For this reason, by solution we mean only the velocity field $U$. In other words, throughout this paper a solution of (1.1) is a vector field $U$ such that there exists some $p$ such that (1.1) is satisfied.

The aim of this paper is to determine the asymptotic behaviour of the solutions at infinity under reasonable assumptions on the forcing $f$. Several authors investigated this problem.

In [1] the authors studied the existence and uniqueness of solutions under a smallness assumption in the critical space $L^{3, \infty}$. Moreover, that article found an explicit asymptotic behaviour of the solutions with a decay as $O\left(\frac{1}{|x|^{2}}\right)$

\footnotetext{
* Corresponding author.

E-mail addresses: agathe.decaster@ens-lyon.fr (A. Decaster), iftimie@ math.univ-lyon1.fr (D. Iftimie).
} 
provided that $\mathbb{P} \Delta^{-1} f$ is bounded by $C /\left(1+|x|^{2}\right)$, where $\mathbb{P}$ is the Leray projector. More precisely they showed the following expansion for the solution:

$$
U(x)=\mathbb{P} \Delta^{-1} f(x)+m(x): \int_{\mathbb{R}^{3}} U \otimes U+O\left(\frac{\ln |x|}{|x|^{3}}\right) \quad \text { as }|x| \rightarrow \infty
$$

where $m(x)$ is an explicit function homogeneous of degree -2 and smooth outside 0 . Observe that $\mathbb{P} \Delta^{-1}$ is a convolution operator whose kernel is a homogeneous function of degree -1 . Therefore, if $f$ is sufficiently decaying at infinity, the condition that $\left|\mathbb{P} \Delta^{-1} f\right| \leqslant C /\left(1+|x|^{2}\right)$ imposed in [1] holds true if and only if $\int_{\mathbb{R}^{3}} f=0$.

But since all terms in the expansion (1.2) are $O\left(\frac{1}{|x|^{2}}\right)$, it excludes all solutions which are homogeneous of degree -1 . In particular it excludes the very important case of Landau solutions. The Landau solutions were introduced by Landau in [10] and they are given by the explicit formula

$$
v_{1}^{c}(x)=2 \frac{c|x|^{2}-2 x_{1}|x|+c x_{1}^{2}}{|x|\left(c|x|-x_{1}\right)^{2}}, \quad v_{2}^{c}(x)=2 \frac{x_{2}\left(c x_{1}-|x|\right)}{|x|\left(c|x|-x_{1}\right)^{2}}, \quad v_{3}^{c}(x)=2 \frac{x_{3}\left(c x_{1}-|x|\right)}{|x|\left(c|x|-x_{1}\right)^{2}}
$$

with pressure

$$
p(x)=4 \frac{c x_{1}-|x|}{|x|\left(c|x|-x_{1}\right)^{2}} .
$$

They verify (1.1) with forcing $f=\beta \delta$ where

$$
\beta=\frac{8 \pi c}{3\left(c^{2}-1\right)}\left(2+6 c^{2}-3 c\left(c^{2}-1\right) \log \left(\frac{c+1}{c-1}\right)\right)
$$

and $\delta$ is the Dirac mass in 0 (see [4]). It was even shown by Šverák [12] that all homogeneous solutions of (1.1) on $\mathbb{R}^{3} \backslash\{0\}$ with vanishing forcing are the Landau solutions.

It appears then that the relevant asymptotic behaviour at infinity of the solutions of (1.1) with forcing sufficiently decaying at infinity should rather be of order $O(1 /|x|)$. And indeed, it was shown in [11] that small solutions of the stationary incompressible Navier-Stokes equations in an exterior domain of $\mathbb{R}^{3}$ behave like $v(x)+o(1 /|x|)$ where $v$ is some unknown vector field homogeneous of degree -1 . Moreover, Korolev and Šverák [9] observed that the asymptotic profile $v$ must be a Landau solution. More precisely, they proved that if $U$ is small and verifies (1.1) with $f=0$ in the exterior of a ball with no boundary conditions required, then there exists $a$ such that $U=v^{a}+o(1 /|x|)$ as $|x| \rightarrow \infty$.

Let us also mention the work [5] where the authors study the stationary Navier-Stokes flow around a rotating body. They obtain again that the asymptotic behaviour of the solution is given by a Landau solution when the speed of rotation of the body is sufficiently small. In [8], the authors prove that the asymptotic behaviour as $|x| \rightarrow \infty$ of time-periodic solutions is also given by a Landau solution.

Since the relevant asymptotic behaviour at infinity is homogeneous of degree -1 and since the forcing corresponding to a velocity homogeneous of degree -1 is homogeneous of degree -3 , it makes sense to study the asymptotic behaviour of the solutions of (1.1) with a forcing whose asymptotic behaviour at infinity is homogeneous of degree -3 .

Let $\alpha \in(0,1)$ be fixed once and for all. We will assume in the rest of this paper that the forcing term is of the form

$$
f=\phi f_{0}+f_{1}
$$

where

- $f_{0}$ is homogeneous of degree -3 , locally bounded on $\mathbb{R}^{3} \backslash\{0\}$;

- we have that $\left|f_{1}(x)\right| \leqslant C /(1+|x|)^{3+\alpha}$ for some constant $C$;

- $\phi \in C^{\infty}\left(\mathbb{R}^{3} ;[0,1]\right)$ is a radial cut-off function such that $\phi(x)=0$ for $|x| \leqslant 1 / 2$ and $\phi(x)=1$ for $|x| \geqslant 1$.

The questions that we ask ourselves are the following. Under what additional hypothesis on $f_{0}$ and $f_{1}$ there exists a solution $U$ of (1.1) such that $|U(x)| \leqslant C /|x|$ for some constant $C$ ? When such a solution exists, how does it behave at infinity? In short, we give the following answers. If such a $U$ exists then necessarily 


$$
\int_{\mathbb{S}^{2}} f_{0}=0
$$

And vice versa, if the above condition is satisfied and if $f_{0}, f_{1}$ are sufficiently small then there exists a unique $U$ which is small and bounded like $O(1 /|x|)$. Moreover, we have the following asymptotic behaviour at infinity for the solution:

$$
U(x)=U_{0}(x)+O\left(|x|^{-1-\alpha}\right),
$$

where $U_{0}$ is the only small solution of the Navier-Stokes equation with forcing term $f_{0}+\left(\int_{\mathbb{R}^{3}} f_{1}\right) \delta$ which is homogeneous of degree -1 .

The precise results and the notation will be given in the next section. In Section 3 we prove a necessary condition for the existence of $O(1 /|x|)$ solutions. We show next in Section 4 the existence and uniqueness of homogeneous solutions. We prove our main result in Section 5. Finally, in Section 6 we extend our results to exterior domains.

\section{Main results and notation}

We introduce the following function space for $a>0$ :

$$
X_{a}=\left\{f \in L_{l o c}^{1}\left(\mathbb{R}^{3} \backslash\{0\}\right) ;|f(x)| \leqslant \frac{C}{|x|^{a}}\right\}
$$

with norm

$$
\|f\|_{X_{a}}=\sup _{x \in \mathbb{R}^{3} \backslash\{0\}}|x|^{a}|f(x)|
$$

We recall now some results on homogeneous distributions that can be found in the book of Hörmander [7, Section 3.2]. Let $v \in \mathscr{D}^{\prime}\left(\mathbb{R}^{3} \backslash\{0\}\right)$ be a distribution homogeneous of degree -3 . There exists a constant $S(v)$ such that the following relation holds true:

$$
\langle v, \varphi\rangle=S(v) \int_{0}^{\infty} \frac{\varphi(r)}{r} d r
$$

for all radial test functions $\varphi \in C_{0}^{\infty}\left(\mathbb{R}^{3} \backslash\{0\}\right)$. We define the integral of $v$ over the unit sphere to be the constant $S(v)$ :

$$
\int_{\mathbb{S}^{2}} v \equiv S(v)
$$

This definition is justified by the fact that the relation above holds true when $v$ is a continuous function as a consequence of the Fubini formula in polar coordinates.

The following result on extensions of homogeneous distributions is stated in [7, Theorem 3.2.4].

Proposition 2.1. (See [7].) Let $g \in \mathscr{D}^{\prime}\left(\mathbb{R}^{3} \backslash\{0\}\right)$ be homogeneous of degree -3. There exists a distribution $h \in \mathscr{D}^{\prime}\left(\mathbb{R}^{3}\right)$ homogeneous of degree -3 such that $\left.h\right|_{\mathbb{R}^{3} \backslash\{0\}}=g$ if and only if $\int_{\mathbb{S}^{2}} g=0$. Moreover, if in addition $g$ is a locally bounded function and $\int_{\mathbb{S}^{2}} g=0$ then all such distributions $h$ are given by

$$
h=\operatorname{pv}(g)+C \delta
$$

where $C$ is an arbitrary constant and the principal value of $g$ is defined by

$$
\operatorname{pv}(g) \in \mathscr{D}^{\prime}\left(\mathbb{R}^{3}\right): \quad\langle\operatorname{pv}(g), \varphi\rangle=\lim _{\varepsilon \rightarrow 0} \int_{|x|>\varepsilon} g \varphi \quad \forall \varphi \in \mathscr{D}\left(\mathbb{R}^{3}\right) .
$$


In what follows we will work with integrals of functions that decay like $1 /|x|^{3}$ at infinity or have local singularities at the origin like $1 /|x|^{3}$. We define such integrals in the principal value sense:

$$
\operatorname{pv} \int_{\mathbb{R}^{3}} h=\lim _{\substack{\varepsilon \rightarrow 0 \\ R \rightarrow \infty}} \int_{\varepsilon<|x|<R} h
$$

provided of course that the limit exists.

We recall now the following well-known (and easy) lemma.

Lemma 2.2. (See [3].) Let $X$ be a Banach space and $B: X \times X \rightarrow X$ a bilinear map. Assume that for all $x_{1}, x_{2} \in X$ one has

$$
\left\|B\left(x_{1}, x_{2}\right)\right\|_{X} \leqslant \eta\left\|x_{1}\right\|_{X}\left\|x_{2}\right\|_{X} .
$$

Then for all $y \in X$ satisfying $4 \eta\|y\|_{X}<1$, the equation

$$
x=y+B(x, x),
$$

has a solution $x \in X$ satisfying and uniquely defined by the condition

$$
\|x\|_{X} \leqslant 2\|y\|_{X} .
$$

The proof of this lemma also shows that $x=\lim _{k \rightarrow \infty} x_{k}$ where the approximate solutions $x_{k}$ are defined by $x_{0}=y$ and $x_{k}=y+B\left(x_{k-1}, x_{k-1}\right)$. Moreover $\left\|x_{k}\right\|_{X} \leqslant 2\|y\|_{X}$ for all $k$.

We denote by $\mathbb{P}$ the Leray projector, i.e. the $L^{2}$ orthogonal projection on the subspace of divergence free vector fields. Applying $\mathbb{P}$ to (1.1) and inverting the laplacian we obtain the following equivalent formulation of (1.1) where there is no pressure:

$$
U=\mathbb{P} \Delta^{-1} \operatorname{div}(U \otimes U)-\mathbb{P} \Delta^{-1} f .
$$

In order to state our main theorem, we first need to establish a result of existence and uniqueness for homogeneous solutions.

Theorem 2.3. Let $f_{0} \in X_{3}$ be homogeneous of degree - 3. If the equations (1.1) on $\mathbb{R}^{3} \backslash\{0\}$ with forcing $f_{0}$ admit a solution $U_{0}$ such that $U_{0} \in X_{1}$ and $U_{0}$ is homogeneous of degree -1 , then necessarily

$$
\int_{\mathbb{S}^{2}} f_{0}=0
$$

and there exists a constant vector $\gamma \in \mathbb{R}^{3}$ such that $U_{0}$ verifies (1.1) on $\mathbb{R}^{3}$ with forcing $\mathrm{pv}\left(f_{0}\right)+\gamma \delta$.

Conversely, assume that $\gamma \in \mathbb{R}^{3}$ and $f_{0} \in X_{3}$ is homogeneous of degree -3 and that (2.3) is satisfied. Then $\mathbb{P} \Delta^{-1} \mathrm{pv}\left(f_{0}\right)$ is well-defined in the principal value sense and there exists $\varepsilon_{1}$ such that if

$$
\left\|f_{0}\right\|_{L^{\infty}\left(\mathbb{S}^{2}\right)}+|\gamma|<\varepsilon_{1}
$$

then there exists a unique solution $U_{0} \in X_{1}$ of (2.2) on $\mathbb{R}^{3}$ with forcing $\mathrm{pv}\left(f_{0}\right)+\gamma \delta$ such that $\left\|U_{0}\right\|_{X_{1}} \leqslant \varepsilon_{1}$. Moreover, $U_{0}$ is homogeneous of degree -1 .

We are now able to state our main theorem:

Theorem 2.4. Let $f$ be as in (1.3). If there is a solution $U$ of (1.1) which belongs to $X_{1}$, then (2.3) must hold true.

Conversely, assume that (2.3) holds true. There exists $\varepsilon_{2}>0$ such that if

$$
\left\|f_{0}\right\|_{L^{\infty}\left(\mathbb{S}^{2}\right)}+\left\|f_{1}\right\|_{X_{0} \cap X_{3+\alpha}} \leqslant \varepsilon_{2}
$$

then the equation (2.2) admits a unique small solution $U \in X_{1}$. Moreover, this solution has the following asymptotic behaviour at infinity:

$$
U(x)=U_{0}(x)+O\left(|x|^{-1-\alpha}\right) \quad \text { as }|x| \rightarrow \infty
$$


where $U_{0}$ is the only -1 homogeneous solution of the Navier-Stokes equation with forcing term $\mathrm{pv}\left(f_{0}\right)+\left(\mathrm{pv} \int_{\mathbb{R}^{3}} f\right) \delta$ obtained in Theorem 2.3.

\section{A necessary condition}

The aim of this section is to prove that if the asymptotic behaviour at infinity of the forcing is homogeneous of degree -3 , then a necessary condition for the existence of $O(1 /|x|)$ solutions is that the asymptotic part of the forcing has vanishing integral on the unit sphere. More precisely, we prove the following result.

Proposition 3.1. Let $R_{0}>0$ and $U$ be a divergence free vector field defined on $\Omega_{0}=\left\{x ;|x|>R_{0}\right\}$ such that $|U(x)| \leqslant$ $C_{0} /|x|$ for some constant $C_{0}$. Let $f_{0}$ and $f_{1}$ be vector valued distributions such that $f_{0} \in \mathscr{D}^{\prime}\left(\mathbb{R}^{3} \backslash\{0\}\right)$ is homogeneous of degree -3 and $f_{1}$ is a (vector valued) bounded Radon measure on $\Omega_{0}$. If there exists some $p \in \mathscr{D}^{\prime}\left(\Omega_{0}\right)$ such that

$$
-\Delta U+(U \cdot \nabla) U+\nabla p=f_{0}+f_{1} \quad \text { in } \mathscr{D}^{\prime}\left(\Omega_{0}\right)
$$

then

$$
\int_{\mathbb{S}^{2}} f_{0}=0
$$

Proof. Let us choose two radial functions $\varphi_{1}$ and $\varphi_{2}$ such that:

- $\varphi_{1} \in C^{\infty}\left(\mathbb{R}^{3} ;[0,1]\right)$ such that $\varphi_{1}(x)=0$ for all $|x| \leqslant R_{0}+1$ and $\varphi_{1}(x)=1$ for all $|x| \geqslant R_{0}+2$.

- $\varphi_{2} \in C_{0}^{\infty}\left(\mathbb{R}^{3} ;[0,1]\right), \varphi_{2}(x)=1$ for all $|x| \leqslant 1$ and $\varphi_{2}(x)=0$ for all $|x| \geqslant 2$.

We define $\varphi^{R}(x)=\varphi_{2}(x / R) \varphi_{1}(x)$ and we observe that $\varphi^{R} \in C_{0}^{\infty}\left(B_{R_{0}}^{c}\right)$. We apply the curl operator to (3.1) and we multiply by the test vector field $V_{R} \equiv \varphi^{R}\left(\begin{array}{c}0 \\ x_{3} \\ -x_{2}\end{array}\right)$ to obtain that

$$
-\left\langle\Delta \operatorname{curl} U, V_{R}\right\rangle+\left\langle\operatorname{curl} \operatorname{div}(U \otimes U), V_{R}\right\rangle=\left\langle\operatorname{curl}\left(f_{0}+f_{1}\right), V_{R}\right\rangle
$$

which implies that

$$
-\left\langle U, \operatorname{curl} \Delta V_{R}\right\rangle-\left\langle U \otimes U, \nabla \operatorname{curl} V_{R}\right\rangle=\left\langle f_{0}+f_{1}, \operatorname{curl} V_{R}\right\rangle .
$$

Let us denote

$$
X=\left(\begin{array}{c}
0 \\
x_{3} \\
-x_{2}
\end{array}\right) \quad \text { and } \quad e_{1}=\left(\begin{array}{l}
1 \\
0 \\
0
\end{array}\right)
$$

We have that

$$
\begin{aligned}
\operatorname{curl} \Delta V_{R} & =\Delta\left(\nabla \varphi^{R} \times X+\varphi^{R} \operatorname{curl} X\right) \\
& =\Delta\left(\nabla \varphi^{R} \times X-2 \varphi^{R} e_{1}\right) \\
& =\nabla \Delta \varphi^{R} \times X+2 \sum_{i=1}^{3} \partial_{i} \nabla \varphi^{R} \times \partial_{i} X-2 \Delta \varphi^{R} e_{1} \\
& =\nabla \Delta \varphi^{R} \times X+2 \partial_{1} \nabla \varphi^{R}-4 \Delta \varphi^{R} e_{1} .
\end{aligned}
$$

Observe next that

$$
\nabla \varphi^{R}(x)=\frac{1}{R} \nabla \varphi_{2}(x / R) \varphi_{1}(x)+\varphi_{2}(x / R) \nabla \varphi_{1}(x)=\frac{1}{R} \nabla \varphi_{2}(x / R)+\nabla \varphi_{1}(x)
$$

if $R \geqslant R_{0}+2$. Therefore, if $R \geqslant R_{0}+2$, 


$$
\begin{aligned}
\operatorname{curl} \Delta V_{R}= & \frac{1}{R^{3}} \nabla \Delta \varphi_{2}(x / R) \times X+\frac{2}{R^{2}} \partial_{1} \nabla \varphi_{2}(x / R)-\frac{4}{R^{2}} \Delta \varphi_{2}(x / R) e_{1} \\
& +\nabla \Delta \varphi_{1} \times X+2 \partial_{1} \nabla \varphi_{1}-4 \Delta \varphi_{1} e_{1} .
\end{aligned}
$$

Clearly

$$
\left|\left\langle U, \frac{1}{R^{3}} \nabla \Delta \varphi_{2}(x / R) \times X\right\rangle\right| \leqslant \frac{C}{R^{3}} \int\left|\nabla \Delta \varphi_{2}(x / R)\right|=C\left\|\nabla \Delta \varphi_{2}\right\|_{L^{1}}
$$

and similarly

$$
\left|\left\langle U, \frac{2}{R^{2}} \partial_{1} \nabla \varphi_{2}(x / R)-\frac{4}{R^{2}} \Delta \varphi_{2}(x / R) e_{1}\right\rangle\right| \leqslant C\left\|\nabla^{2} \varphi_{2} /|x|\right\|_{L^{1}} .
$$

We infer that

$$
\left\langle U, \operatorname{curl} \Delta V_{R}\right\rangle=O(1) \quad \text { as } R \rightarrow \infty .
$$

It can be shown in a similar fashion that

$$
\left\langle U \otimes U, \nabla \operatorname{curl} V_{R}\right\rangle=O(1) \quad \text { as } R \rightarrow \infty .
$$

We deduce from (3.2) that $\left\langle f_{0}+f_{1}, \operatorname{curl} V_{R}\right\rangle$ must also be bounded as $R \rightarrow \infty$. But

$$
\begin{aligned}
\left\langle f_{0}+f_{1}, \operatorname{curl} V_{R}\right\rangle & =\left\langle f_{0}+f_{1},-2 \varphi^{R} e_{1}+\nabla \varphi^{R} \times X\right\rangle \\
& =-2\left\langle\left(f_{0}\right)_{1}, \varphi^{R}\right\rangle-2\left\langle\left(f_{1}\right)_{1}, \varphi^{R}\right\rangle+\left\langle f_{0}, \nabla \varphi^{R} \times X\right\rangle+\left\langle f_{1}, \nabla \varphi^{R} \times X\right\rangle \\
& \equiv I_{1}+I_{2}+I_{3}+I_{4} .
\end{aligned}
$$

Recall that $\nabla \varphi^{R}$ can be expressed as in (3.3). By homogeneity, we observe that $I_{3}$ does not depend on $R$. Because $\varphi^{R}$ and $\nabla \varphi^{R} \times X$ are uniformly bounded and because $f_{1}$ is a bounded Radon measure, we have that $I_{2}$ and $I_{4}$ are bounded. We infer that $I_{1}$ must be bounded as $R \rightarrow \infty$. Since $f_{0}$ is homogeneous of degree -3 and $\varphi^{R}$ is radial, we have from (2.1) that

$$
\int_{\mathbb{R}^{3}}\left(f_{0}\right)_{1} \varphi^{R}=\int_{0}^{\infty} \frac{\varphi^{R}(r)}{r} d r \int_{\mathbb{S}^{2}}\left(f_{0}\right)_{1}
$$

But $\varphi^{R}$ is nonnegative and $\varphi^{R}(r)=1$ for all $r \in\left[R_{0}+2, R\right]$ so

$$
\int_{0}^{\infty} \frac{\varphi^{R}(r)}{r} d r \geqslant \int_{R_{0}+2}^{R} \frac{\varphi^{R}(r)}{r} d r \geqslant \int_{R_{0}+2}^{R} \frac{1}{r} d r=\ln (R)-\ln \left(R_{0}+2\right) .
$$

We conclude that for $\left\langle\left(f_{0}\right)_{1}, \varphi^{R}\right\rangle$ to be bounded it is necessary to have that $\int_{\mathbb{S}^{2}}\left(f_{0}\right)_{1}=0$. The same argument can be applied to the other components of $f_{0}$, so we finally deduce that

$$
\int_{\mathbb{S}^{2}} f_{0}=0
$$

must hold true. This completes the proof of the proposition.

\section{Homogeneous solutions}

In this section, we prove Theorem 2.3.

Assume first that there exists $U_{0} \in X_{1}$ homogeneous of degree -1 and some pressure $p_{0}$ such that

$$
-\Delta U_{0}+\operatorname{div}\left(U_{0} \otimes U_{0}\right)+\nabla p_{0}=f_{0} \quad \text { in } \mathbb{R}^{3} \backslash\{0\} .
$$

Proposition 3.1 immediately implies that (2.3) must hold true.

We prove now that there exists a constant vector $\gamma \in \mathbb{R}^{3}$ such that $U_{0}$ verifies (1.1) on $\mathbb{R}^{3}$ with forcing $\operatorname{pv}\left(f_{0}\right)+\gamma \delta$. 
Since $\left|U_{0}\right| \leqslant C /|x|$ we observe that $U_{0} \in L_{\text {loc }}^{1}\left(\mathbb{R}^{3}\right)$. Therefore $U_{0}$ defines a distribution of $\mathscr{D}^{\prime}\left(\mathbb{R}^{3}\right)$ which is homogeneous of degree -1 . Taking the laplacian of this distribution implies that $\Delta U_{0}$ admits an extension to a distribution on the whole $\mathbb{R}^{3}$ which is homogeneous of degree -3 . Similarly, $U_{0} \otimes U_{0}$ is bounded by $C /|x|^{2}$ so it belongs to $L_{\text {loc }}^{1}\left(\mathbb{R}^{3}\right) \subset \mathscr{D}^{\prime}\left(\mathbb{R}^{3}\right)$ so $\operatorname{div}\left(U_{0} \otimes U_{0}\right)$ also admits an extension to a distribution on the whole $\mathbb{R}^{3}$ which is homogeneous of degree -3 . Let us define $\bar{f}_{0}$ as follows

$$
-\Delta U_{0}+\operatorname{div}\left(U_{0} \otimes U_{0}\right)=\bar{f}_{0} .
$$

We have that $\bar{f}_{0}$ is a distribution on $\mathbb{R}^{3}$ which is homogeneous of degree -3 . Clearly

$$
\operatorname{curl} \bar{f}_{0}=\operatorname{curl}\left(-\Delta U_{0}+\operatorname{div}\left(U_{0} \otimes U_{0}\right)\right)=\operatorname{curl} f_{0} \quad \text { in } \mathscr{D}^{\prime}\left(\mathbb{R}^{3} \backslash\{0\}\right)
$$

so curl $\bar{f}_{0}-\operatorname{curl} \operatorname{pv}\left(f_{0}\right)$ is a distribution in $\mathscr{D}^{\prime}\left(\mathbb{R}^{3}\right)$, homogeneous of degree -4 and supported in the origin. Therefore, there exist three constant vectors $A_{1}, A_{2}, A_{3}$ such that

$$
\operatorname{curl}\left(\bar{f}_{0}-\operatorname{pv}\left(f_{0}\right)\right)=A_{1} \partial_{1} \delta+A_{2} \partial_{2} \delta+A_{3} \partial_{3} \delta \quad \text { in } \mathscr{D}^{\prime}\left(\mathbb{R}^{3}\right) .
$$

Because of the identity div curl $=0$ we infer that

$$
\operatorname{div}\left(A_{1} \partial_{1} \delta+A_{2} \partial_{2} \delta+A_{3} \partial_{3} \delta\right)=0
$$

so

$$
\sum_{i, j=1}^{3} a_{i j} \partial_{i j} \delta=0
$$

where $a_{i j}$ are the components of the matrix $A$ whose columns are $A_{1}, A_{2}, A_{3}$. Because the derivatives of the Dirac mass are linearly independent we infer that the matrix $A$ must be skew-symmetric. Therefore there exists some vector $\gamma=\left(\begin{array}{l}\gamma_{1} \\ \gamma_{2} \\ \gamma_{3}\end{array}\right)$ such that

$$
A=\left(\begin{array}{ccc}
0 & \gamma_{3} & -\gamma_{2} \\
-\gamma_{3} & 0 & \gamma_{1} \\
\gamma_{2} & -\gamma_{1} & 0
\end{array}\right) .
$$

Then it can be easily checked that

$$
A_{1} \partial_{1} \delta+A_{2} \partial_{2} \delta+A_{3} \partial_{3} \delta=\operatorname{curl}(\gamma \delta)
$$

so we deduce from (4.2) that

$$
\operatorname{curl}\left(\bar{f}_{0}-\operatorname{pv}\left(f_{0}\right)-\gamma \delta\right)=0 \quad \text { in } \mathscr{D}^{\prime}\left(\mathbb{R}^{3}\right) .
$$

We infer that there exists some distribution $p \in \mathscr{D}^{\prime}\left(\mathbb{R}^{3}\right)$ such that

$$
\bar{f}_{0}-\mathrm{pv}\left(f_{0}\right)-\gamma \delta=\nabla p
$$

so equation (4.1) can be rewritten under the form

$$
-\Delta U_{0}+\operatorname{div}\left(U_{0} \otimes U_{0}\right)=\operatorname{pv}\left(f_{0}\right)+\gamma \delta+\nabla p \quad \text { in } \mathscr{D}^{\prime}\left(\mathbb{R}^{3}\right) .
$$

This means that $U_{0}$ verifies the Navier-Stokes equation on $\mathbb{R}^{3}$ with forcing $\mathrm{pv}\left(f_{0}\right)+\gamma \delta$.

Assume now that $\int_{\mathbb{S}^{2}} f_{0}=0$. Then by Proposition 2.1 we have that $\operatorname{pv}\left(f_{0}\right)$ is well defined and homogeneous of degree -3 . It is not difficult to see that the Fourier transform of $\operatorname{pv}\left(f_{0}\right)$ is a bounded function, so $\mathbb{P} \Delta^{-1} \operatorname{pv}\left(f_{0}\right)$ can be easily defined in Fourier space. However, we need some estimates for $\mathbb{P} \Delta^{-1} \mathrm{pv}\left(f_{0}\right)$ in the space $X_{1}$ so we prefer to avoid using the Fourier transform and prove directly that $\mathbb{P} \Delta^{-1} \mathrm{pv}\left(f_{0}\right)$ is well defined and estimate it in $X_{1}$.

Let us recall that $\mathbb{P} \Delta^{-1}$ is a convolution operator with kernel given by the following matrix (see [6]):

$$
G(x)=-\frac{1}{8 \pi}\left(\frac{I_{3}}{|x|}+\frac{x \otimes x}{|x|^{3}}\right)
$$

where $I_{3}$ is the identity matrix.

We prove next that the convolution $G * f_{0}$ is well defined. 
Lemma 4.1. The convolution $G * f_{0}$ is well defined in the principal value sense:

$$
G * f_{0}(x)=\operatorname{pv} \int_{\mathbb{R}^{3}} G(x-y) f_{0}(y) d y
$$

and we have the bound

$$
\left\|G * f_{0}\right\|_{X_{1}} \leqslant C\left\|f_{0}\right\|_{X_{3}}=C\left\|f_{0}\right\|_{L^{\infty}\left(\mathbb{S}^{2}\right)}
$$

for some universal constant $C$.

Proof. The existence of $G * f_{0}$ in the principal value sense follows from the estimates below. We decompose

$$
\begin{aligned}
\mathrm{pv} \int_{\mathbb{R}^{3}} G(x-y) f_{0}(y) d y= & \int_{|y|>2|x|} G(x-y) f_{0}(y) d y+\int_{2|x|>|y|>|x| / 2} G(x-y) f_{0}(y) d y \\
& +\mathrm{pv} \int_{|x| / 2>|y|} G(x-y) f_{0}(y) d y \\
= & \int_{|y|>2|x|} G(x-y) f_{0}(y) d y+\int_{2|x|>|y|>|x| / 2} G(x-y) f_{0}(y) d y \\
& +\int_{|x| / 2>|y|}(G(x-y)-G(x)) f_{0}(y) d y \\
\equiv & I_{1}+I_{2}+I_{3}
\end{aligned}
$$

where we used that $f_{0}$ has vanishing mean on the unit sphere so

$$
\mathrm{pv} \int_{|x| / 2>|y|} f_{0}(y) d y=0 .
$$

We bound now each of these terms. Observe first that

$$
\begin{aligned}
\left|I_{1}\right| & =\left|\int_{|y|>2|x|} G(x-y) f_{0}(y) d y\right| \\
& \leqslant \int_{|y|>2|x|} \frac{C\left\|f_{0}\right\|_{L^{\infty}\left(\mathbb{S}^{2}\right)}}{|x-y||y|^{3}} d y \\
& \leqslant C\left\|f_{0}\right\|_{L^{\infty}\left(\mathbb{S}^{2}\right)} \int_{|y|>2|x|} \frac{1}{|y|^{4}} d y \leqslant \frac{C\left\|f_{0}\right\|_{L^{\infty}\left(\mathbb{S}^{2}\right)} .}{|x|} .
\end{aligned}
$$

Next

$$
\begin{aligned}
\left|I_{2}\right| & =\left|\int_{2|x|>|y|>|x| / 2} G(x-y) f_{0}(y) d y\right| \\
& \leqslant \int_{2|x|>|y|>|x| / 2} \frac{C\left\|f_{0}\right\|_{L^{\infty}\left(\mathbb{S}^{2}\right)}}{|x-y||y|^{3}} d y \\
& \leqslant \frac{C\left\|f_{0}\right\|_{L^{\infty}\left(\mathbb{S}^{2}\right)}}{|x|^{3}} \int_{|x-y|<3|x|} \frac{1}{|x-y|} d y \leqslant \frac{C\left\|f_{0}\right\|_{L^{\infty}\left(\mathbb{S}^{2}\right)}}{|x|} .
\end{aligned}
$$


We estimate now $I_{3}$. By the mean value theorem we have that $G(x-y)-G(x)=y \cdot \nabla G(\xi)$ for some $\xi \in[x, x-y]$. If $|y|<|x| / 2$ then we can bound

$$
|G(x-y)-G(x)| \leqslant|y| \sup _{\xi \in[x, x-y]}|\nabla G(\xi)| \leqslant C|y| \sup _{\xi \in[x, x-y]} \frac{1}{|\xi|^{2}} \leqslant C \frac{|y|}{|x|^{2}} .
$$

Therefore

$$
\left|I_{3}\right|=\left|\int_{|x| / 2>|y|}(G(x-y)-G(x)) f_{0}(y) d y\right| \leqslant \frac{C\left\|f_{0}\right\|_{L^{\infty}\left(\mathbb{S}^{2}\right)}}{|x|^{2}} \int_{|x| / 2>|y|} \frac{1}{|y|^{2}} d y \leqslant \frac{C\left\|f_{0}\right\|_{L^{\infty}\left(\mathbb{S}^{2}\right)}}{|x|} .
$$

Putting together the above estimates completes the proof of the lemma.

Once this lemma is proved, it is not difficult to finish the proof of Theorem 2.3. Indeed, we have that $G * \delta=G \in X_{1}$ so $G *\left(\operatorname{pv}\left(f_{0}\right)+\gamma \delta\right) \in X_{1}$. Therefore $\mathbb{P} \Delta^{-1}\left(\mathrm{pv}\left(f_{0}\right)+\gamma \delta\right)$ is well defined, belongs to $X_{1}$ and we have the estimate

$$
\left\|\mathbb{P} \Delta^{-1}\left(\operatorname{pv}\left(f_{0}\right)+\gamma \delta\right)\right\|_{X_{1}} \leqslant C\left\|f_{0}\right\|_{L^{\infty}\left(\mathbb{S}^{2}\right)}+C|\gamma| .
$$

To show the existence and the uniqueness of a small solution $U_{0}$ it suffices to appeal to the following result from [1, Theorems 2.2 and 3.1]:

Theorem 4.2. (See [1].) There exists an absolute constant $\varepsilon_{0}>0$ with the following property. If $f$ is such that $\mathbb{P} \Delta^{-1} f \in X_{1}$ and

$$
\left\|\mathbb{P} \Delta^{-1} f\right\|_{X_{1}}<\varepsilon_{0}
$$

then there exists a unique solution $U \in X_{1}$ of (2.2) such that

$$
\|U\|_{X_{1}} \leqslant 2\left\|\mathbb{P} \Delta^{-1} f\right\|_{X_{1}} .
$$

Because of (4.4), we can apply the above theorem and deduce that if $\left\|f_{0}\right\|_{L^{\infty}\left(\mathbb{S}^{2}\right)}+|\gamma|$ is sufficiently small, then there exists a unique small solution $U_{0}$ of (2.2) with forcing $\mathrm{pv}\left(f_{0}\right)+\gamma \delta$. Moreover, we have that

$$
\left\|U_{0}\right\|_{X_{1}} \leqslant C\left\|f_{0}\right\|_{L^{\infty}\left(\mathbb{S}^{2}\right)}+C|\gamma| \text {. }
$$

It remains to show that $U_{0}$ is homogeneous of degree -1 . This follows from a standard scaling argument. By homogeneity, $\operatorname{pv}\left(f_{0}\right)+\gamma \delta$ is invariant through the scaling $f \mapsto \lambda^{3} f(\lambda x)$. Moreover, the $X_{1}$ norm of $\mathbb{P} \Delta^{-1} f$ is also invariant through this scaling. But if $U_{0}$ solves the Navier-Stokes equations with forcing $f$ then $U_{0, \lambda}(x)=\lambda U_{0}(\lambda x)$ also solves the Navier-Stokes equations with forcing $\lambda^{3} f(\lambda x)$. By uniqueness of solutions, we must have that $U_{0, \lambda}=$ $U_{0}$ for all $\lambda>0$. Therefore the solution $U_{0}$ must be homogeneous of degree -1 .

\section{Asymptotic behaviour for general solutions}

In this section we prove Theorem 2.4.

The necessity of the condition (2.3) for the existence of $U \in X_{1}$ is a consequence of Proposition 3.1.

We assume now that the condition (2.3) is verified. It can be proved exactly like in Lemma 4.1 that $\mathbb{P} \Delta^{-1}\left(\phi f_{0}\right)=$ $G *\left(\phi f_{0}\right)$ is well defined and belongs to $X_{1}$ and that

$$
\left\|\mathbb{P} \Delta^{-1}\left(\phi f_{0}\right)\right\|_{X_{1}} \leqslant C\left\|f_{0}\right\|_{L^{\infty}\left(\mathbb{S}^{2}\right)}
$$

for some universal constant $C$. Since $f_{1} \in X_{0} \cap X_{3+\alpha}$, we know from [1, relation (3.1)] that $\mathbb{P} \Delta^{-1} f_{1} \in X_{1}$ and

$$
\left\|\mathbb{P} \Delta^{-1} f_{1}\right\|_{X_{1}} \leqslant C\left\|f_{1}\right\|_{X_{0} \cap X_{3+\alpha}} .
$$

We infer that $\mathbb{P} \Delta^{-1} f \in X_{1}$ and

$$
\left\|\mathbb{P} \Delta^{-1} f\right\|_{X_{1}} \leqslant C\left(\left\|f_{0}\right\|_{L^{\infty}\left(\mathbb{S}^{2}\right)}+\left\|f_{1}\right\|_{X_{0} \cap X_{3+\alpha}}\right) \leqslant C \varepsilon_{2} .
$$

Let us recall now the following result, see [1, Lemma 3.6]: 
Lemma 5.1. Let $U_{1} \in X_{1+\beta}$ and $U_{2} \in X_{1+\gamma}$ with $\beta, \gamma \geqslant 0$ and $\beta+\gamma<1$. We have that $\mathbb{P} \Delta^{-1} \operatorname{div}\left(U_{1} \otimes U_{2}\right) \in X_{1+\gamma+\beta}$ and

$$
\left\|\mathbb{P} \Delta^{-1} \operatorname{div}\left(U_{1} \otimes U_{2}\right)\right\|_{X_{1+\gamma+\beta}} \leqslant C\left\|U_{1}\right\|_{X_{1+\beta}}\left\|U_{2}\right\|_{X_{1+\gamma}} .
$$

Therefore the application $X_{1} \times X_{1} \ni\left(U_{1}, U_{2}\right) \mapsto \mathbb{P} \Delta^{-1} \operatorname{div}\left(U_{1} \otimes U_{2}\right) \in X_{1}$ is bilinear and continuous. Applying Lemma 2.2 we deduce that if $\varepsilon_{2}$ is sufficiently small, then there exists a unique solution $U$ of (2.2) which is small in $X_{1}$. We know from the proof of Lemma 2.2 that the solution $U$ is obtained as the limit of the approximate solutions defined by

$$
U^{0}=-\mathbb{P} \Delta^{-1} f, \quad U^{k+1}=\mathbb{P} \Delta^{-1} \operatorname{div}\left(U^{k} \otimes U^{k}\right)-\mathbb{P} \Delta^{-1} f .
$$

Moreover, we have that $\left\|U^{k}\right\|_{X_{1}} \leqslant C \varepsilon_{2}$ for all $k$.

Let $U_{0}$ be the only -1 homogeneous solution of the Navier-Stokes equation with forcing term $\mathrm{pv}\left(f_{0}\right)+\left(\mathrm{pv} \int_{\mathbb{R}^{3}} f\right) \delta$ as obtained in Theorem 2.3. Let us define $v=U-U_{0}$. To complete the proof of Theorem 2.4 we need to show that $v \in X_{1+\alpha}$. We have that $v$ solves the following equation:

$$
v=\mathbb{P} \Delta^{-1} \operatorname{div}\left(v \otimes v+v \otimes U_{0}+U_{0} \otimes v\right)-\mathbb{P} \Delta^{-1} f_{2}
$$

where

$$
f_{2}=(\phi-1) f_{0}+f_{1}-\left(\int_{\mathbb{R}^{3}} f_{1}\right) \delta .
$$

We used above that $\int_{\mathbb{S}^{2}} f_{0}=0$ and that $\phi$ is radial to deduce that pv $\int_{\mathbb{R}^{3}} \phi f_{0}=0$ so $\int_{\mathbb{R}^{3}} f_{1}=\mathrm{pv} \int_{\mathbb{R}^{3}} f$. Because $U$ is the limit of the approximate solutions $U^{k}$, we have that $v$ is the limit of the approximate solutions $v_{k}=U^{k}-U_{0}$ which solve

$$
v_{0}=-\mathbb{P} \Delta^{-1} f_{2}, \quad v_{k+1}=\mathbb{P} \Delta^{-1} \operatorname{div}\left(v_{k} \otimes v_{k}+v_{k} \otimes U_{0}+U_{0} \otimes v_{k}\right)-\mathbb{P} \Delta^{-1} f_{2} .
$$

We have that

$$
\left\|v_{k}\right\|_{X_{1}} \leqslant C \varepsilon_{2} \quad \forall k .
$$

Indeed, we observed above that $\left\|U^{k}\right\|_{X_{1}} \leqslant C \varepsilon_{2}$ for all $k$ and we also know from the proof of Theorem 2.3 that $\left\|U_{0}\right\|_{X_{1}} \leqslant C \varepsilon_{2}$ (see relation (4.5)).

We will show by induction that there exists some small $\varepsilon_{3}$ such that if $\varepsilon_{2}$ is small enough then $v_{k} \in X_{1+\alpha}$ for all $k$ and

$$
\left\|v_{k}\right\|_{X_{1+\alpha}} \leqslant \varepsilon_{3} .
$$

The first step is to prove this bound for $k=0$.

Lemma 5.2. We have that

$$
\left\|\mathbb{P} \Delta^{-1} f_{2}\right\|_{X_{1+\alpha}} \leqslant C\left(\left\|f_{0}\right\|_{L^{\infty}\left(\mathbb{S}^{2}\right)}+\left\|f_{1}\right\|_{X_{3+\alpha}}\right) .
$$

Proof. Recall that $\mathbb{P} \Delta^{-1}$ is a convolution operator with the kernel $G$ defined in (4.3). We write

$$
\begin{aligned}
\mathbb{P} \Delta^{-1}\left(\phi f_{0}+f_{1}\right)(x)= & \int_{\mathbb{R}^{3}} G(x-y) \cdot\left(\phi(y) f_{0}(y)+f_{1}(y)\right) d y \\
= & \operatorname{pv} \int_{\mathbb{R}^{3}} G(x-y) f_{0}(y) d y+G(x) \cdot \int_{\mathbb{R}^{3}} f_{1}+\mathrm{pv} \int_{\mathbb{R}^{3}} G(x-y) \cdot(\phi-1)(y) f_{0}(y) d y \\
& +\int_{\mathbb{R}^{3}}(G(x-y)-G(x)) \cdot f_{1}(y) d y
\end{aligned}
$$




$$
\begin{aligned}
= & G *\left(f_{0}+\int_{\mathbb{R}^{3}} f_{1} \delta\right)(x)+\int_{|y| \leqslant 1}(G(x-y)-G(x)) \cdot(\phi-1)(y) f_{0}(y) d y \\
& +\int_{\mathbb{R}^{3}}(G(x-y)-G(x)) \cdot f_{1}(y) d y
\end{aligned}
$$

We deduce that

$$
\begin{aligned}
\left|\mathbb{P} \Delta^{-1}\left(f_{2}\right)(x)\right| \leqslant & \left|\int_{|y| \leqslant 1}(G(x-y)-G(x)) \cdot(\phi-1) f_{0}(y) d y\right|+\left|\int_{\mathbb{R}^{3}}(G(x-y)-G(x)) \cdot f_{1}(y) d y\right| \\
\leqslant & \left|\int_{|y| \leqslant \min (|x| / 2,1)}(G(x-y)-G(x)) \cdot(\phi-1) f_{0}(y) d y\right| \\
& +\left|\int_{|x| / 2 \leqslant|y| \leqslant 1}(G(x-y)-G(x)) \cdot(\phi-1) f_{0}(y) d y\right| \\
& +\left|\int_{|y| \leqslant|x| / 2}(G(x-y)-G(x)) \cdot f_{1}(y) d y\right|+\left|\int_{|y| \geqslant|x| / 2}(G(x-y)-G(x)) \cdot f_{1}(y) d y\right| \\
\equiv & I_{1}+I_{2}+I_{3}+I_{4},
\end{aligned}
$$

where we assume that $I_{2}=0$ if $|x|>2$. We estimate now each of these terms. First

$$
\begin{aligned}
I_{1} & \leqslant \int_{|y| \leqslant \min (|x| / 2,1)}|G(x-y)-G(x)|\left|(\phi-1) f_{0}(y)\right| d y \\
& \leqslant C\left\|f_{0}\right\|_{L^{\infty}\left(\mathbb{S}^{2}\right)} \int_{|y| \leqslant \min (|x| / 2,1)} \frac{|\nabla G(\zeta(x, y)) \cdot y|}{|y|^{3}} d y
\end{aligned}
$$

where $\zeta(x, y)$ is between $x$ and $x-y$, so $1 /|\zeta|^{2} \leqslant C /|x|^{2}$

$$
\begin{aligned}
& \leqslant C\left\|f_{0}\right\|_{L^{\infty}\left(\mathbb{S}^{2}\right)} \int_{|y| \leqslant \min (|x| / 2,1)} \frac{1}{|x|^{2}|y|^{2}} d y \\
& \leqslant \frac{C\left\|f_{0}\right\|_{L^{\infty}\left(\mathbb{S}^{2}\right)}}{|x|^{2}} \min (|x| / 2,1) \\
& \leqslant \frac{C\left\|f_{0}\right\|_{L^{\infty}\left(\mathbb{S}^{2}\right)}}{|x|^{1+\alpha}}
\end{aligned}
$$

Next, if $|x| \leqslant 2$ then we can bound $I_{2}$ exactly as in Lemma 4.1 to obtain that

$$
\left|I_{2}\right| \leqslant \frac{C\left\|f_{0}\right\|_{L^{\infty}\left(\mathbb{S}^{2}\right)}}{|x|} \leqslant \frac{C\left\|f_{0}\right\|_{L^{\infty}\left(\mathbb{S}^{2}\right)}}{|x|^{1+\alpha}} .
$$

We can estimate $I_{3}$ as follows:

$$
\begin{aligned}
I_{3} & \leqslant \int_{|y| \leqslant|x| / 2}|G(x-y)-G(x)|\left|f_{1}(y)\right| d y \\
& \leqslant C\left\|f_{1}\right\|_{X_{3+\alpha}} \int_{|y| \leqslant|x| / 2} \frac{|\nabla G(\zeta) \cdot y|}{|y|^{3+\alpha}} d y
\end{aligned}
$$


where $\zeta(x, y)$ is between $x$ and $x-y$, so $1 /|\zeta|^{2} \leqslant C /|x|^{2}$

$$
\begin{aligned}
& \leqslant \frac{C\left\|f_{1}\right\|_{X_{3+\alpha}}}{|x|^{2}} \int_{|y| \leqslant|x| / 2} \frac{1}{|y|^{2+\alpha}} d y \\
& \leqslant \frac{C\left\|f_{1}\right\|_{X_{3+\alpha}}}{|x|^{1+\alpha}} .
\end{aligned}
$$

Finally

$$
\begin{aligned}
I_{4} & \leqslant \int_{|y| \geqslant|x| / 2}|G(x-y)-G(x)|\left|f_{1}(y)\right| d y \\
& \leqslant \int_{|x| / 2 \leqslant|y| \leqslant 2|x|}|G(x-y)|\left|f_{1}(y)\right| d y+\int_{|y| \geqslant 2|x|}|G(x-y)|\left|f_{1}(y)\right| d y+\int_{|y| \geqslant|x| / 2}|G(x)|\left|f_{1}(y)\right| d y \\
& \leqslant C\left\|f_{1}\right\|_{X_{3+\alpha}}\left(\frac{1}{|x|^{3+\alpha}} \int_{|x| / 2 \leqslant|y| \leqslant 2|x|} \frac{1}{|x-y|} d y+\int_{|y| \geqslant 2|x|} \frac{1}{|y|^{4+\alpha}} d y+\frac{1}{|x|} \int_{|y| \geqslant|x| / 2} \frac{1}{|y|^{3+\alpha}} d y\right) \\
& \leqslant \frac{C\left\|f_{1}\right\|_{X_{3+\alpha}}}{|x|^{1+\alpha}} .
\end{aligned}
$$

This completes the proof of the lemma.

We now go back to the proof of relation (5.2). From Lemma 5.2 we deduce that

$$
\left\|v_{0}\right\|_{X_{1+\alpha}} \leqslant C \varepsilon_{2} \text {. }
$$

We impose the condition $C \varepsilon_{2} \leqslant \varepsilon_{3}$ so that (5.2) is verified for $k=0$. Suppose that we have proved (5.2) for $v_{k}$, we want to prove it for $v_{k+1}$. We use relation (5.1) and Lemma 5.1 to estimate

$$
\begin{aligned}
\left\|v_{k+1}\right\|_{X_{1+\alpha}} & \leqslant C\left(\left\|v_{k}\right\|_{X_{1+\alpha}}\left\|v_{k}\right\|_{X_{1}}+\left\|v_{k}\right\|_{X_{1+\alpha}}\left\|U_{0}\right\|_{X_{1}}\right)+\left\|v_{0}\right\|_{X_{1+\alpha}} \\
& \leqslant C \varepsilon_{2}\left\|v_{k}\right\|_{X_{1+\alpha}}+C \varepsilon_{2} \\
& \leqslant C \varepsilon_{2}\left(1+\varepsilon_{3}\right) .
\end{aligned}
$$

Clearly if $\varepsilon_{2}$ is sufficiently small then we can choose some $\varepsilon_{3}$ such that $C \varepsilon_{2}\left(1+\varepsilon_{3}\right) \leqslant \varepsilon_{3}$. For such a choice of $\varepsilon_{2}$ and $\varepsilon_{3}$, relation (5.2) holds true for all $k$. Therefore it must hold true for the limit $v$. So $v \in X_{1+\alpha}$ and this completes the proof of Theorem 2.4.

\section{The exterior domain case}

In this section, we prove the following extension of Theorem 2.4 to exterior domains.

Theorem 6.1. Let $R>0$ and consider $f, U$ and $p$ be defined for $|x|>R$. Assume that $U \in X_{1}$ and $f=f_{0}+f_{1}$ with $f_{0} \in X_{3}$ homogeneous of degree -3 and $f_{1} \in X_{3+\alpha}$. We assume moreover that

$$
-\Delta U+(U \cdot \nabla) U+\nabla p=f, \quad \operatorname{div} U=0 \quad \text { in } \quad\{|x|>R\},
$$

that condition (2.3) holds true and that

$$
\int_{|x|=R_{1}} U \cdot x=0
$$

for all $R_{1}>R$. There exists $\varepsilon>0$ such that, if

$$
\left\|f_{0}\right\|_{X_{3}}+\left\|f_{1}\right\|_{X_{3+\alpha}}+\|U\|_{X_{1}} \leqslant \varepsilon
$$


then there exists some small constant vector $m_{0}$ such that $U$ admits the following asymptotic behaviour:

$$
U(x)=U_{0}(x)+O\left(\frac{1}{|x|^{1+\alpha}}\right) \quad \text { as }|x| \rightarrow \infty
$$

where $U_{0}$ is the only -1 homogeneous solution of the Navier-Stokes equation with forcing term $\mathrm{pv}\left(f_{0}\right)+m_{0} \delta$ obtained in Theorem 2.3. Moreover, we can express $m_{0}$ under the following form

$$
m_{0}=\int_{|x|>R_{1}} f_{1}+\int_{|x|=R_{1}}\left[-\partial_{\nu} U+(U \cdot v) U+p \nu\right] \quad \forall R_{1}>R
$$

where $v=x /|x|$.

Remark 6.2. The condition (6.1) is natural. It is automatically satisfied if $U$ verifies the Navier-Stokes equations together with homogeneous Dirichlet boundary conditions in an exterior domain $\Omega$. This can be easily checked by integrating the relation $\operatorname{div} U=0$ on $\Omega \cap\left\{|x|<R_{1}\right\}$. Moreover, a similar integration by parts shows that the integral in (6.1) is proportional to $R_{1}$ so it suffices to assume (6.1) for only one $R_{1}$.

To prove Theorem 6.1, we proceed as in [9]. We extend $U$ to the whole space, we study the additional forcing term that appears and we apply Theorem 2.4.

Let $\Omega=\{|x|>R\}$. First, let us observe that $U$ and $p$ are more regular than stated:

Lemma 6.3. We have that $(U, p) \in W_{l o c}^{2, q}(\Omega) \times W_{l o c}^{1, q}(\Omega)$ for any $1<q<\infty$.

Proof of the lemma. The proof follows from the following interior regularity result for the stationary Stokes equation proved in [13]:

Theorem 6.4. (See [13].) Let $\Omega$ be a domain in $\mathbb{R}^{3}$, and $B_{1} \subset B_{2}$ be concentric balls of radii $R$ and $2 R$, strictly contained in $\Omega$. Let $1<q<\infty$, and $f \in W_{l o c}^{-1, q}(\Omega)$. If $(U, p) \in W_{l o c}^{1, q}(\Omega) \times L_{l o c}^{q}(\Omega)$ is a pair of solutions of the stationary Stokes system with forcing term $f$ in $\Omega$ (without any boundary condition), then

$$
\|U\|_{W^{1, q}\left(B_{1}\right)}+\inf _{c \in \mathbb{R}}\|p-c\|_{L^{q}\left(B_{1}\right)} \leqslant C\left(\|U\|_{L^{1}\left(B_{2} \backslash B_{1}\right)}+\|f\|_{W^{-1, q}\left(B_{2}\right)}\right) .
$$

This theorem implies the following regularity result: if $U \in L_{l o c}^{1}(\Omega)$ verifies the Stokes equation in $\Omega$ with forcing $f \in$ $W_{l o c}^{-1, q}(\Omega)$, then $U \in W_{l o c}^{1, q}(\Omega)$. Indeed, we can approximate $U$ by using cut-off and convolution with an approximation of the identity. The regularized velocity verifies the Stokes equation on a sub-domain of $\Omega$ (where the cut-off function is 1), so by Theorem 6.4 it will be bounded in $W_{l o c}^{1, q}$ of that sub-domain. So its limit $U$ will also belong to $W_{l o c}^{1, q}$ of that sub-domain. Since the sub-domain can be an arbitrary bounded sub-domain of $\Omega$, we infer that $U \in W_{l o c}^{1, q}(\Omega)$.

Going back to the proof of the lemma we observe that $f \in W_{l o c}^{-1, q}(\Omega)$ and $\operatorname{div}(U \otimes U) \in W_{l o c}^{-1, q}(\Omega)$ for any $1<q<\infty$. Since we have

$$
\begin{aligned}
-\Delta U+\nabla p & =f-\operatorname{div}(U \otimes U) \\
\operatorname{div} U & =0
\end{aligned}
$$

in $\Omega$, we can apply the above mentioned regularity result to obtain that $U \in W_{l o c}^{1, q}(\Omega)$ for any $1<q<\infty$. Then we can derive these equations and we get:

$$
\begin{aligned}
-\Delta\left(\partial_{j} U\right)+\nabla\left(\partial_{j} p\right) & =\partial_{j} f+\operatorname{div}\left(\partial_{j}(U \otimes U)\right) \\
\operatorname{div}\left(\partial_{j} U\right) & =0
\end{aligned}
$$

for any $j \in\{1,2,3\}$. Again $\partial_{j} f \in W_{l o c}^{-1, q}(\Omega)$ for any $1<q<\infty$. Moreover, since $U \in L_{\text {loc }}^{\infty}(\Omega) \cap W_{\text {loc }}^{1, q}(\Omega)$, we have that $\partial_{j}(U \otimes U)=\partial_{j} U \otimes U+U \otimes \partial_{j} U \in L_{l o c}^{q}(\Omega)$ for any $1<q<\infty$. So $\operatorname{div} \partial_{j}(U \otimes U) \in W_{l o c}^{-1, q}(\Omega)$ for any 
$1<q<\infty$. Reapplying the same regularity result implies that $U \in W_{l o c}^{2, q}(\Omega)$ for any $1<q<\infty$. From the equation we also get that $\nabla p \in L_{l o c}^{q}(\Omega)$ so $p \in W_{l o c}^{1, q}(\Omega)$ for any $1<q<\infty$. This completes the proof of the lemma.

Let $R<R_{0}<R_{1}$ and consider a radial cut-off function $\eta \in C^{\infty}\left(\mathbb{R}^{3},[0,1]\right)$ such that $\eta=0$ on $B\left(0, R_{0}\right)$ and $\eta=1$ on $B\left(0, R_{1}\right)^{c}$. We define the following extension of the solution $(U, p)$ :

$$
\begin{aligned}
& \widetilde{U}=U, \quad \tilde{p}=p \quad \text { on } B\left(0, R_{1}\right)^{c} \\
& \widetilde{U}=\eta U+v, \quad \tilde{p}=\eta p \quad \text { on } B\left(0, R_{1}\right)
\end{aligned}
$$

where we extended $\eta U$ and $\eta p$ with zero values for $|x| \leqslant R$. The vector field $v$ is constructed in such a manner as to ensure that $\widetilde{U} \in L^{\infty} \cap X_{1}$ and $\operatorname{div} \widetilde{U}=0$ everywhere. Therefore $v$ must verify the following problem:

$$
\begin{aligned}
\operatorname{div} v & =-U \cdot \nabla \eta \text { in } B\left(0, R_{1}\right) \\
v & =0 \text { on } \partial B\left(0, R_{1}\right)
\end{aligned}
$$

This problem has many solutions, and a way to find one with good estimates is given by the Bogovskiĭ operators, see [2]. In particular, we have the following result:

Theorem 6.5. (See [2].) Let $g \in W_{0}^{k, q}(B)$ where $B$ is a ball and $k \in \mathbb{N}, 1<q<\infty$. Assume that $\int_{B} g=0$. Then there exists a solution $V \in W_{0}^{k+1, q}(B)$ of the equation $\operatorname{div} V=g$, with the following estimate:

$$
\|V\|_{W^{k+1, q}(B)} \leqslant C(q, k, B)\|g\|_{W^{k, q}(B)} .
$$

We have that

$$
\int_{B\left(0, R_{1}\right)} U \cdot \nabla \eta=\int_{S\left(0, R_{1}\right)} U \cdot v \eta-\int_{B\left(0, R_{1}\right)} \eta \operatorname{div} U=\int_{S\left(0, R_{1}\right)} U \cdot v=0
$$

where we used (6.1). Moreover, because $U \in W_{l o c}^{2, q}(\Omega)$ for any $1<q<\infty$ we have that $U \cdot \nabla \eta \in W^{2, q}$ for any $1<q<\infty$. Using Theorem 6.5 we infer that there exists $v \in W_{0}^{3, q}\left(B\left(0, R_{1}\right)\right)$ for all $1<q<\infty$ a solution of (6.3). We extend $v$ to the whole space $\mathbb{R}^{3}$ by setting $v=0$ for $|x|>R_{1}$ so that $v \in W^{3, q}\left(\mathbb{R}^{3}\right)$.

We observe now that the extension $(\widetilde{U}, \widetilde{p})$ verifies the following stationary Navier-Stokes equation in the whole space:

$$
-\Delta \widetilde{U}+(\widetilde{U} \cdot \nabla) \widetilde{U}+\nabla \widetilde{p}=\eta f+F, \quad \operatorname{div} \widetilde{U}=0
$$

where

$$
F=-\Delta v-\Delta \eta U-2 \nabla U \cdot \nabla \eta+\operatorname{div}\left(\eta U \otimes v+\eta v \otimes U+v \otimes v+\eta^{2} U \otimes U\right)-\eta \operatorname{div}(U \otimes U)+p \nabla \eta
$$

is compactly supported in $B\left(0, R_{1}\right)$.

Given that $U \in W_{l o c}^{2, q}, p \in W_{l o c}^{1, q}$ and $v \in W^{3, q}$ for all $1<q<\infty$, one can use Sobolev embeddings to deduce that $F$ is bounded. From the estimates of Theorems 6.4 and 6.5 we know that the $W_{l o c}^{2, q}$ norms of $U$ and the $W^{3, q}$ norm of $v$ can be bounded in terms of the $L_{l o c}^{\infty}$ norms of $U$ and $f$. Because these norms are assumed to be small, we conclude that $F$ is bounded, small and compactly supported. Moreover, $\widetilde{U}$ is also small in $X_{1}$. We can therefore apply Theorem 2.4 to $(6.4)$ to deduce that $\widetilde{U}$ has the following asymptotic behaviour:

$$
\widetilde{U}=U_{0}+O\left(\frac{1}{|x|^{1+\alpha}}\right)
$$

where $U_{0}$ is the only -1 homogeneous solution of the Navier-Stokes equation with forcing term $\operatorname{pv}\left(f_{0}\right)+m_{0} \delta$ obtained in Theorem 2.3 and

$$
m_{0}=\int_{\mathbb{R}^{3}} \eta f_{1}+\int_{\mathbb{R}^{3}} F
$$


It remains to prove relation (6.2). We integrate relation (6.4) on the ball $B\left(0, R_{1}\right)$ and use the Stokes formula to obtain that

$$
\int_{B\left(0, R_{1}\right)}\left(\eta f_{1}+F\right)=\int_{B\left(0, R_{1}\right)} \operatorname{div}\left(-\nabla \tilde{U}+\widetilde{U} \otimes \tilde{U}+\widetilde{p} I_{3}\right)=\int_{S\left(0, R_{1}\right)}\left[-\partial_{\nu} U+(U \cdot v) U+p v\right]
$$

Since $F$ is compactly supported in $B\left(0, R_{1}\right)$ we have that

$$
\int_{B\left(0, R_{1}\right)} F=\int_{\mathbb{R}^{3}} F
$$

so relation (6.2) follows immediately.

\section{Conflict of interest statement}

There is no conflict of interest.

\section{Acknowledgements}

The authors have been partially funded by the ANR project Dyficolti ANR-13-BS01-0003-01. This work was supported by the LABEX MILYON (ANR-10-LABX-0070) of Universite de Lyon, within the program "Investissements d'Avenir" (ANR-11-IDEX-0007) operated by the French National Research Agency (ANR).

\section{References}

[1] C. Bjorland, L. Brandolese, D. Iftimie, M.E. Schonbek, Lp-solutions of the steady-state Navier-Stokes equations with rough external forces, Commun. Partial Differ. Equ. 36 (2) (2011) 216-246.

[2] M.E. Bogovskiľ, Solution of the first boundary value problem for an equation of continuity of an incompressible medium, Dokl. Akad. Nauk SSSR 248 (5) (1979) 1037-1040.

[3] M. Cannone, Ondelettes, Paraproduits et Navier-Stokes, Diderot Éditeur, Arts et Sciences, 1995.

[4] M. Cannone, G. Karch, Smooth or singular solutions to the Navier-Stokes system?, J. Differ. Equ. 197 (2) (2004) $247-274$.

[5] R. Farwig, T. Hishida, Asymptotic profile of steady Stokes flow around a rotating obstacle, Manuscr. Math. 136 (3-4) (2011) $315-338$.

[6] G.P. Galdi, An Introduction to the Mathematical Theory of the Navier-Stokes Equations. Vol. I, Linearised Steady Problems, Springer, 1997.

[7] L. Hormander, The Analysis of Linear Partial Differential Operators I: Distribution Theory and Fourier Analysis, 2nd revised edition, SpringerVerlag, Berlin, Heidelberg, 2003, GmbH \& Co. K.

[8] K. Kang, H. Miura, T.-P. Tsai, Asymptotics of small exterior Navier-Stokes flows with non-decaying boundary data, Commun. Partial Differ. Equ. 37 (10) (2012) 1717-1753.

[9] A. Korolev, V. Šverák, On the large-distance asymptotics of steady state solutions of the Navier-Stokes equations in 3D exterior domains, Ann. Inst. Henri Poincaré, Anal. Non Linéaire 28 (2) (2011) 303-313.

[10] L. Landau, A new exact solution of Navier-Stokes equations, C. R. (Dokl.) Acad. Sci. URSS 43 (1944) 286-288.

[11] S.A. Nazarov, K. Pileckas, On steady Stokes and Navier-Stokes problems with zero velocity at infinity in a three-dimensional exterior domain, Kyoto J. Math. 40 (3) (2000) 475-492.

[12] V. Šverák, On Landau's solutions of the Navier-Stokes equations, J. Math. Sci. 179 (1) (2011) 208-228.

[13] V. Šverák, T.-P. Tsai, On the spatial decay of 3-D steady-state Navier-Stokes flows, Commun. Partial Differ. Equ. 25 (11-12) (2000) 2107-2117. 